\title{
Nationwide analysis of treatment outcomes in children and adolescents routinely treated for tuberculosis in the Netherlands
}

\author{
Fajri Gafar (10 ${ }^{1,8}$, Natasha van't Boveneind-Vrubleuskaya ${ }^{2,3,8}$, \\ Onno W. Akkerman ${ }^{4,5}$, Bob Wilffert ${ }^{1,2}$ and Jan-Willem C. Alffenaar ${ }^{2,6,7}$
}

@ERSpublications

High success rates for TB treatment were achieved in children and adolescents in the Netherlands. To further optimise care in this population, several risk factors particularly associated with mortality and loss to follow-up have been identified. http://bit.ly/2lLJRTC

Cite this article as: Gafar F, van't Boveneind-Vrubleuskaya N, Akkerman OW, et al. Nationwide analysis of treatment outcomes in children and adolescents routinely treated for tuberculosis in the Netherlands. Eur Respir J 2019; 54: 1901402 [https://doi.org/10.1183/13993003.01402-2019].

\section{ABSTRACT}

Background: As a vulnerable population, children and adolescents with tuberculosis (TB) are faced with many challenges, even those who live in low TB incidence countries. We aimed to evaluate factors associated with TB treatment outcomes allowing more focused interventions to support this population once diagnosed.

Methods: A retrospective cohort study using a nationwide surveillance database was performed in children and adolescents (aged 0-18 years) treated for TB in the Netherlands from 1993 to 2018. Logistic regression analyses were used to estimate adjusted odds ratios (aOR) for associated factors of mortality and loss to follow-up (LTFU).

Results: Among 3253 eligible patients with known outcomes, 94.4\% (95.9\% children and 92.8\% adolescents) were cured or completed treatment, $0.7 \%$ died during treatment and $4.9 \%$ were LTFU. There were no reported treatment failures. Risk factors of death included children aged 2-4 years (aOR 10.42), central nervous system TB (aOR 5.14), miliary TB (aOR 10.25), HIV co-infection (aOR 8.60), re-treated $\mathrm{TB}$ cases (aOR 10.12) and drug-induced liver injury ( $\mathrm{aOR}$ 6.50). Active case-finding was a protective factor of death (aOR 0.13). Risk factors of LTFU were adolescents aged 15-18 years (aOR 1.91), illegal immigrants (aOR 4.28), urban domicile (aOR 1.59), unknown history of TB contact (aOR 1.99), drugresistant $\mathrm{TB}$ (aOR 2.31), single adverse drug reaction (aOR 2.12), multiple adverse drug reactions (aOR 7.84) and treatment interruption $>14$ days (aOR 6.93). Treatment in recent years (aOR 0.94) and supervision by public health nurses (aOR 0.14 ) were protective factors of LTFU.

Conclusion: Highly successful treatment outcomes were demonstrated in children and adolescents routinely treated for TB. Special attention should be given to specific risk groups to improve treatment outcomes. 


\section{Introduction}

Tuberculosis (TB) is a major global health problem with an estimated 1 million children worldwide developing TB in 2017 [1]. Childhood TB has historically been given low priority in most national programmes because it contributes little to disease transmission. Similarly, adolescents are faced with many challenges as they have been neglected in TB surveillance, even when they suffer a significant burden of the disease [2,3]. Since the World Health Organization (WHO) published 10 key actions in 2013 as the first roadmap for childhood TB [4], significant progress has been made, but gaps still remain, especially in age- and disease-related challenges such as young children (aged $<5$ years), adolescents (aged 10-19 years), TB/HIV co-infection and multidrug-resistant (MDR)-TB. The 2018 WHO roadmap brings new hope of accelerating efforts towards ending TB in children and adolescents by ensuring that they receive high priority in all $\mathrm{TB}$ prevention and control activities [5].

As one of the top 10 causes of death, childhood TB is a silent killer, with the risk of mortality being particularly high in children aged $<5$ years and HIV co-infected children not receiving antiretroviral therapy (ART) [6,7]. In low-incidence countries like the Netherlands, TB elimination requires extensive yet focused screening and prevention as the patients become more concentrated in certain vulnerable and high-risk groups such as the poor, immigrants, asylum seekers, homeless, prisoners, alcohol or drug addicts and people living with HIV/AIDS [8]. Management of childhood and adolescent TB is still a pressing challenge even for low-incidence countries, particularly due to the lack of child-friendly drug formulations, difficulties in diagnosis and treatment of latent $\mathrm{TB}$ infection (LTBI) [9].

A few studies with large cohorts of children, mostly from high-incidence settings in Africa, have reported factors associated with $\mathrm{TB}$ treatment outcomes in children [10-14]. However, most of the variables analysed in these studies were relatively limited to demographic and clinical characteristics. Other potential confounders such as vaccination status, types of case-finding, drug-susceptibility of the TB strains and other clinical-, bacteriological- and treatment-related factors have not been fully evaluated. In addition, related data in both children and adolescents from low-incidence countries are lacking. In this context, our study aimed to evaluate treatment outcomes and associated factors in children and adolescents routinely treated for TB in the Netherlands. This would allow for appropriate interventions to optimise TB care in this vulnerable population.

\section{Methods}

\section{Study design and data sources}

This retrospective cohort study was performed using surveillance data obtained from the Netherlands Tuberculosis Register. The Netherlands Tuberculosis Register is a nationwide database for patients with TB and LTBI, managed by the Dutch National Institute for Public Health and the Environment (RIVM) in collaboration with 25 departments of the Municipal Public Health Services (MPHS) and the Royal Netherlands Tuberculosis Association (KNCV). Since 1993, data on disease notification, demographics, clinical, bacteriological and treatment characteristics are recorded by the MPHS in all TB patients.

\section{Study population}

All children and adolescents (aged 0-18 years) treated for TB between January 1993 and December 2018 were included in this study. Patients in ongoing treatment with incomplete data on treatment outcomes were excluded.

\section{Data collection}

The following individual data with anonymous identifiers were obtained from the Netherlands Tuberculosis Register on May 22 2019: 1) demographics (year of diagnosis, age, sex, native/foreign-born,

Affiliations: ${ }^{1}$ University of Groningen, Groningen Research Institute of Pharmacy, Unit of PharmacoTherapy, -Epidemiology, and -Economics, Groningen, The Netherlands. ${ }^{2}$ University of Groningen, University Medical Center Groningen, Dept of Clinical Pharmacy and Pharmacology, Groningen, The Netherlands. ${ }^{3}$ Dept of Public Health TB Control, Metropolitan Public Health Services, The Hague, The Netherlands. "University of Groningen, University Medical Center Groningen, Dept of Pulmonary Diseases and Tuberculosis, Groningen, The Netherlands. ${ }^{5}$ University of Groningen, University Medical Center Groningen, Tuberculosis Center Beatrixoord, Haren, The Netherlands. 'University of Sydney, Faculty of Medicine and Health, School of Pharmacy, Sydney, Australia. ${ }^{7}$ Westmead Hospital, Sydney, Australia. ${ }^{8}$ Both authors contributed equally.

Correspondence: Fajri Gafar, University of Groningen, Groningen Research Institute of Pharmacy, Unit of PharmacoTherapy, -Epidemiology, and -Economics, Antonius Deusinglaan 1 (room: 3214.0450), 9713 AV Groningen, The Netherlands. E-mail: f.gafarArug.nl 
WHO region of birth, immigrant status and living area); 2) TB notification and clinical characteristics (types of case-finding, history of TB contact, travel history in TB-endemic area, site and localisation of TB, cavitary TB, bacille Calmette-Guérin (BCG) vaccination, TB symptoms, patient's and doctor's delay in diagnosis and treatment, and comorbidity); 3) bacteriological characteristics (acid-fast bacilli smear microscopy, mycobacterial culture and drug susceptibility testing (DST)); and 4) treatment characteristics (previous history of TB/LTBI treatment, daily/intermittent dosing, presence of adverse drug reactions (ADRs), drug-induced liver injury (DILI), treatment interruption $>14$ days, hospitalisation, treatment supervision by public health nurses (PHNs) and implementation of directly observed therapy).

\section{Definitions}

Age was generally divided into two groups: children aged $<15$ years and adolescents aged $15-18$ years. The cut-off of $<15$ years for children was used to be consistent with the age category used for reporting TB surveillance data nationally and by the WHO [1]. The upper age limit of 18 years for adolescent TB was based on the definition used by the WHO European region [15]. Active case-finding was defined as the systematic screening for active TB cases in a predetermined high-risk group for TB, rather than waiting for patients who came on their own to the healthcare system because of TB symptoms (passive case-finding). Pulmonary TB included all forms of TB in the lungs, isolated tracheal or bronchus TB, laryngeal TB and other specified respiratory TB. TB within other locations in the body than the lungs, including mediastinal lymphadenopathy, were classified as extrapulmonary TB (EPTB), which may have involved isolated EPTB or a combination of pulmonary TB and EPTB. Confirmed drug-susceptible TB was defined as a susceptible result of DST for all first-line anti-TB drugs (isoniazid, rifampicin, pyrazinamide and ethambutol), while presumed drug-susceptible TB was defined as patients treated with first-line anti-TB drugs without sufficient information on DST. Patients with DST results of monoresistant, polyresistant, MDR- or extensively drug-resistant (XDR)-TB were classified as confirmed drug-resistant TB. DILI due to anti-TB drugs was defined as an increased level of alanine aminotransferase more than three times the upper limit of normal in the presence of symptoms of hepatotoxicity or more than five times the upper limit of normal in the absence of symptoms. Treatment supervision by PHNs was defined as supportive discussions with patients and their family to provide TB education and identification of obstacles that influence treatment adherence. Directly observed therapy was defined as every dose of anti-TB drugs taken under direct observation for a period of time, provided by either PHNs or other selected third parties such as family members or home nursing services. Operational definitions for all variables are shown in supplementary table S1 $[16,17]$.

\section{Outcomes}

Treatment outcomes (cured, treatment completed, died, treatment failed, lost to follow-up (LTFU), and not evaluated (unknown outcomes)) were defined based on the Dutch national guidelines for TB programmes and generally in accordance with the current WHO guidelines (table 1) $[16,18]$.

\section{Data analysis}

Associations of patient characteristics with mortality and LTFU were evaluated. First, patients who died during treatment were compared to those who were alive at the end of treatment regardless of whether they were cured, completed or failed treatment; this definition excluded LTFU and unknown outcomes. Second, LTFU patients were compared to those who achieved cure or completed treatment with or without evidence of treatment failure; this definition excluded death and unknown outcomes. Given the possibility of selection bias from the exclusion of particular patients in the first and second analyses, additional outcome classification was created assessing patients who achieved cure or completed treatment (favourable) compared to all other outcomes (unfavourable).

Univariate and multivariate logistic regression analyses were used to evaluate the association between candidate variables and treatment outcomes. All variables in the univariate analysis showing a trend towards association with each of the evaluated outcomes, and with a minimum number of 20 patients in any particular group of predictors, were eligible for inclusion in multivariate analysis and were selected using backward elimination. The final multivariate models retained all explanatory variables with a p-value $<0.1$. The Hosmer-Lemeshow test was used to evaluate the goodness of fit of the final models. The performance of the final models was measured by the area under the receiver operating characteristic curve. Crude and adjusted odds ratios (aORs) with 95\% confidence intervals were used to estimate the association between explanatory variables and treatment outcomes. Statistical significance was accepted at $\mathrm{p}<0.05$, whereas $\mathrm{p}$-values of $0.05-0.10$ were considered trends. All data were analysed using SPSS Statistics (version 25.0; IBM, Armonk, NY, USA). 
TABLE 1 Treatment outcome definitions used in this study

Definition for drug-susceptible TB

A patient who had completed a full course of therapy or $\geqslant 80 \%$ of the prescribed doses with a confirmed culture-negative result at the end of treatment

Treatment completed

A patient who had taken all of the prescribed doses or $\geqslant 80 \%$ of them without any information on sputum culture at the end of treatment

Died

Treatment failed

A patient who died for any reason before starting or during the course of treatment

A patient whose sputum culture was positive after 5 months or later during treatment

Lost to follow-up

\section{Not evaluated (unknown)}

\section{Treatment success} treatment interruption for two consecutive months or more; treatment completion of $<80 \%$ of the prescribed doses; treatment incompletion of 6 months within the 9-month treatment period; or treatment incompletion of 9 months within the 12-month treatment period

A patient for whom no treatment outcome lcured, treatment completed, died, treatment failed and LTFU) was assigned in the database; this included cases "transferred out" to another unit (country) with unknown treatment results

The sum of "cured" and "treatment completed"
A patient who met one of the following criteria:

\section{Definition for drug-resistant TB}

A patient who had treatment completed without evidence of failure with three or more consecutive negative cultures taken $\geqslant 30$ days apart after the intensive phase

A patient who had treatment completed without evidence of failure, but no record that three or more consecutive cultures taken $\geqslant 30$ days apart were negative after the intensive phase

A patient who died for any reason during the course of treatment

A patient who met one of the following criteria: lack of conversion by the end of the intensive phase; bacteriological reversion in the continuation phase after conversion to negative; evidence of additional acquired resistance to fluoroquinolone or second-line injectable drugs; or evidence of adverse drug reactions requiring discontinuation of treatment

A patient whose treatment was interrupted for two consecutive months or more

A patient for whom no treatment outcome was assigned in the database; this included cases "transferred out" to another unit (country) with unknown treatment results

The sum of "cured" and "treatment completed"

TB: tuberculosis; LTFU: lost to follow-up. Drug-resistant TB comprised monoresistant TB (resistance to one first-line anti-TB drug only), polyresistant TB (resistance to more than one first-line anti-TB drug other than isoniazid and/or rifampicin), multidrug-resistant (MDR)-TB (resistance to at least both isoniazid and rifampicin), extensively drug-resistant TB (resistance to any fluoroquinolone and to at least one of three second-line injectable drugs (capreomycin, kanamycin and amikacin) in addition to MDR-TB), and rifampicin-resistant TB (resistance to rifampicin with or without resistance to other anti-TB drugs) $[16,18]$.

Ethics

Research approval was granted by the research committee of the Netherlands Tuberculosis Register. As this was a retrospective study using routine data collected anonymously, ethics clearance and individual patient written informed consent were not required under Dutch law.

\section{Results}

During a 26-year period from January 1993 to December 2018, 3442 TB cases in children and adolescents were notified: 46 patients in ongoing treatment were excluded. Of 3396 eligible patients (1764 (51.9\%) children and 1632 (48.1\%) adolescents), 1893 (55.7\%) were male, 2017 (59.4\%) were foreign-born and 1454 (42.8\%) had pulmonary TB. Mycobacterial culture was performed in 2261 (66.6\%) of the eligible patients with 1921 (56.6\%) being culture-positive and 340 (10\%) culture-negative. Out of 1921 patients with culture-confirmed disease, 1610 (83.8\%) had information on species. Of these, $1523(94.6 \%)$ had Mycobacterium tuberculosis and 87 (5.4\%) had other M. tuberculosis complex. None of the patients were identified as nontuberculous mycobacterial infections. Most of the patients $(n=2625,77.3 \%)$ were treated as presumed drug-susceptible TB, $591(17.4 \%)$ as confirmed drug-susceptible $\mathrm{TB}$ and $180(5.3 \%)$ as confirmed drug-resistant TB (table 2). Severe forms of TB (central nervous system (CNS) or miliary TB) were notified in $100(2.9 \%)$ out of 3396 eligible patients: 33 (33\%) had received BCG vaccination and 44 (44\%) were not BCG-vaccinated. Out of 44 severe cases who were not BCG-vaccinated, 23 (52.3\%) were children aged $<5$ years, $15(34.1 \%)$ were children aged $5-14$ years and the remaining six (13.6\%) were adolescents.

By including patients with both known and unknown outcomes, overall success rates of $92.0 \%$ and $88.7 \%$ were shown in children and adolescents, respectively. Known outcomes were recorded in 3253 (95.8\%) out of 3396 eligible patients. Of these, success rates were $95.9 \%$ in children and $92.8 \%$ in adolescents (table 2). 
TABLE 2 Characteristics of children and adolescents treated for tuberculosis (TB) in the Netherlands

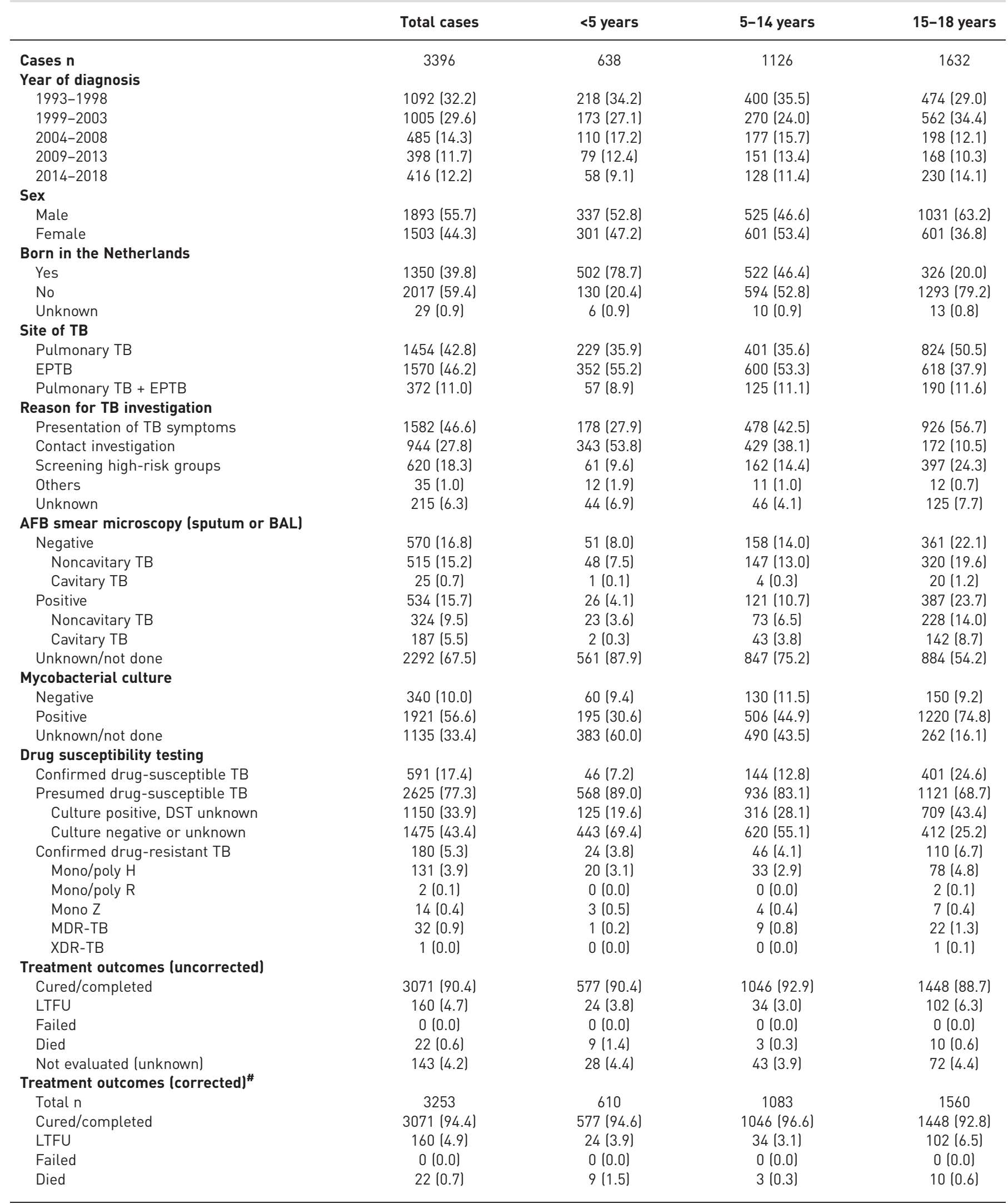

Data are presented as $\mathrm{n}(\%)$, unless otherwise stated. EPTB: extrapulmonary tuberculosis; AFB: acid-fast bacilli; BAL: bronchoalveolar lavage; DST: drug susceptibility testing; H: isoniazid; R: rifampicin; Z: pyrazinamide; MDR: multidrug-resistant; XDR: extensively drug-resistant; LTFU: lost to follow-up. \# : excluded patients with unknown outcomes. 

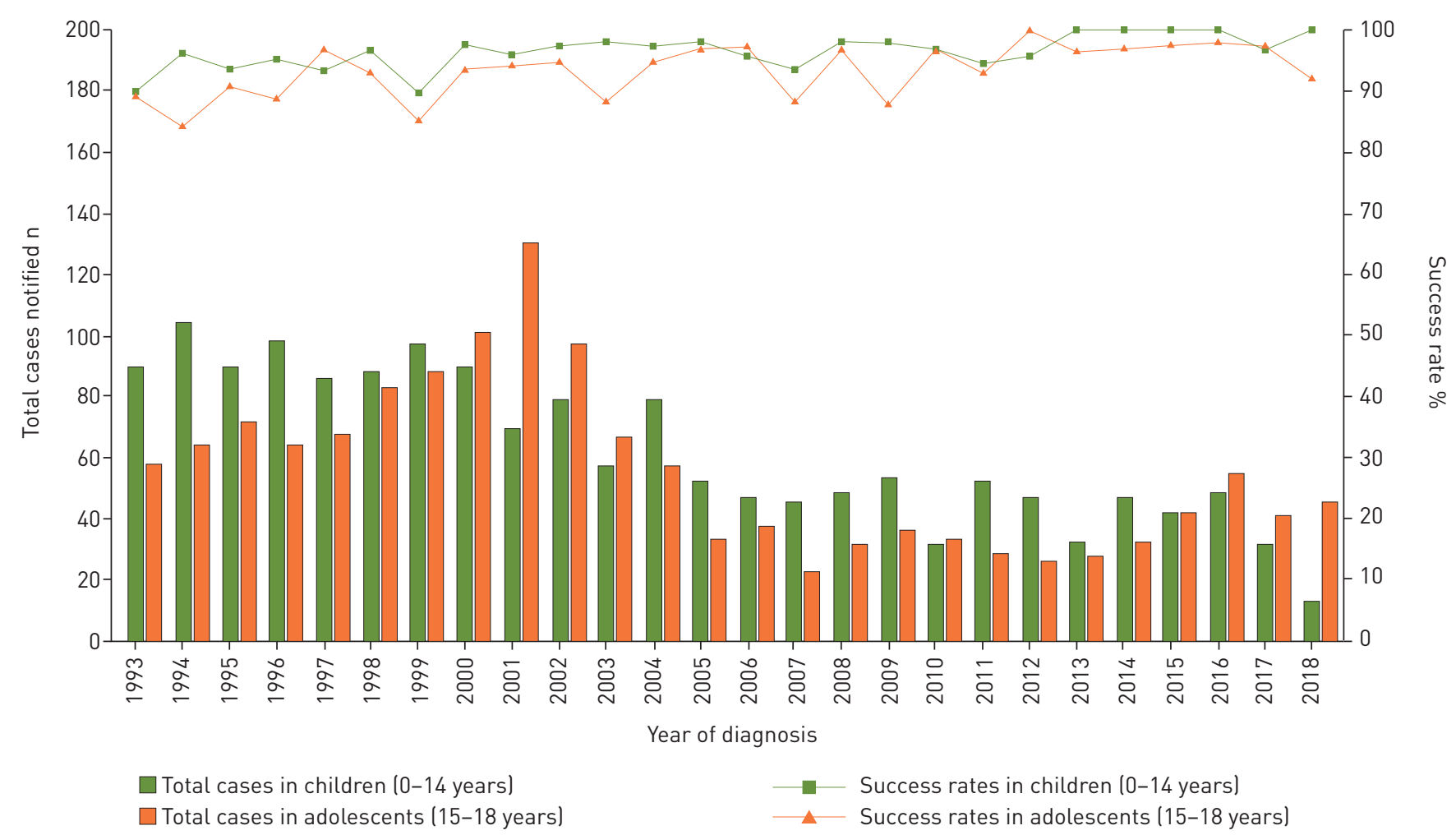

Success rates in children (0-14 years)
Success rates in adolescents (15-18 years)

FIGURE 1 Notified tuberculosis cases and trend of success rates among children and adolescents treated for tuberculosis in the Netherlands, 1993-2018.

Annual success rates in children were constantly $>90 \%$ over the years from 1993 to 2018, and relatively higher compared to adolescents (figure 1). LTFU was higher in adolescents $(n=102,6.5 \%)$ than children $(\mathrm{n}=58,3.4 \%)$. No treatment failure was reported and $22(0.7 \%)$ out of all patients died during treatment (table 2). Case fatality rates for other subpopulations are presented in supplementary table S2.

Our multivariate model showed that children aged 2-4 years had an increased odds of death compared to children aged 5-14 years (aOR 10.42, 95\% CI 2.25-48.36). In addition, positive associations with mortality were shown in patients with CNS TB (aOR 5.14, 95\% CI 1.17-22.62), miliary TB (aOR 10.25, 95\% CI 2.30-45.67), HIV co-infection (aOR 8.60, 95\% CI 1.57-47.24), re-treated TB cases (aOR 10.12, 95\% CI 1.54-66.47) and those who developed DILI during therapy (aOR 6.50, 95\% CI 1.09-38.71). Active case-finding was associated with lower odds of death compared to passive case-finding (aOR 0.13, 95\% CI 0.03-0.66) (table 3). Patients with unknown history of TB contact, unknown BCG status, who experienced TB symptoms or were hospitalised for $\geqslant 1$ week during treatment had a significantly increased odds of death in univariate analysis, but did not remain significant in multivariate analysis (supplementary table S3). Although unknown history of TB contact was not sustained in multivariate analysis as a predictor of mortality, it was significantly associated with higher odds of either patient's delay (aOR 2.36, 95\% CI 1.463.80 ) or doctor's delay (aOR 4.29, 95\% CI 2.48-7.42) compared to known TB contact history, adjusted for age, sex, smear microscopy and sites of TB.

Several factors were associated with higher odds of LTFU, including adolescence (aOR 1.91, 95\% CI 1.252.93), illegal immigrants (aOR 4.28, 95\% CI 1.60-11.42), urban domicile (aOR 1.59, 95\% CI 1.10-2.29), unknown history of TB contact (aOR 1.99, 95\% CI 1.19-3.34), confirmed drug-resistant TB (aOR 2.31, 95\% CI 1.05-5.10), single ADR (aOR 2.12, 95\% CI 1.18-3.83), multiple ADRs (aOR 7.84, 95\% CI 3.5517.33) and treatment interruption lasting $>14$ days (aOR 6.93,95\% CI 2.72-17.63). Treatment in recent years (aOR 0.94, 95\% CI 0.89-0.98) and treatment supervision by PHNs (aOR 0.14, 95\% CI 0.07-0.29) were associated with lower odds of LTFU (table 4). Being male and foreign-born were significantly associated with higher odds of LTFU in univariate analysis, but not found to be statistically significant in multivariate analysis (supplementary table S4). However, our subgroup analysis identified that male foreign-born adolescents had a significantly increased odds of LTFU compared to female foreign-born adolescents (aOR 2.31, 95\% CI 1.30-4.10), adjusted for year of diagnosis, area of living, DST results, presence of ADRs, treatment interruption lasting $>14$ days and treatment supervision by PHNs. 
TABLE 3 Final model for factors associated with mortality in children and adolescents treated for tuberculosis (TB) in the Netherlands

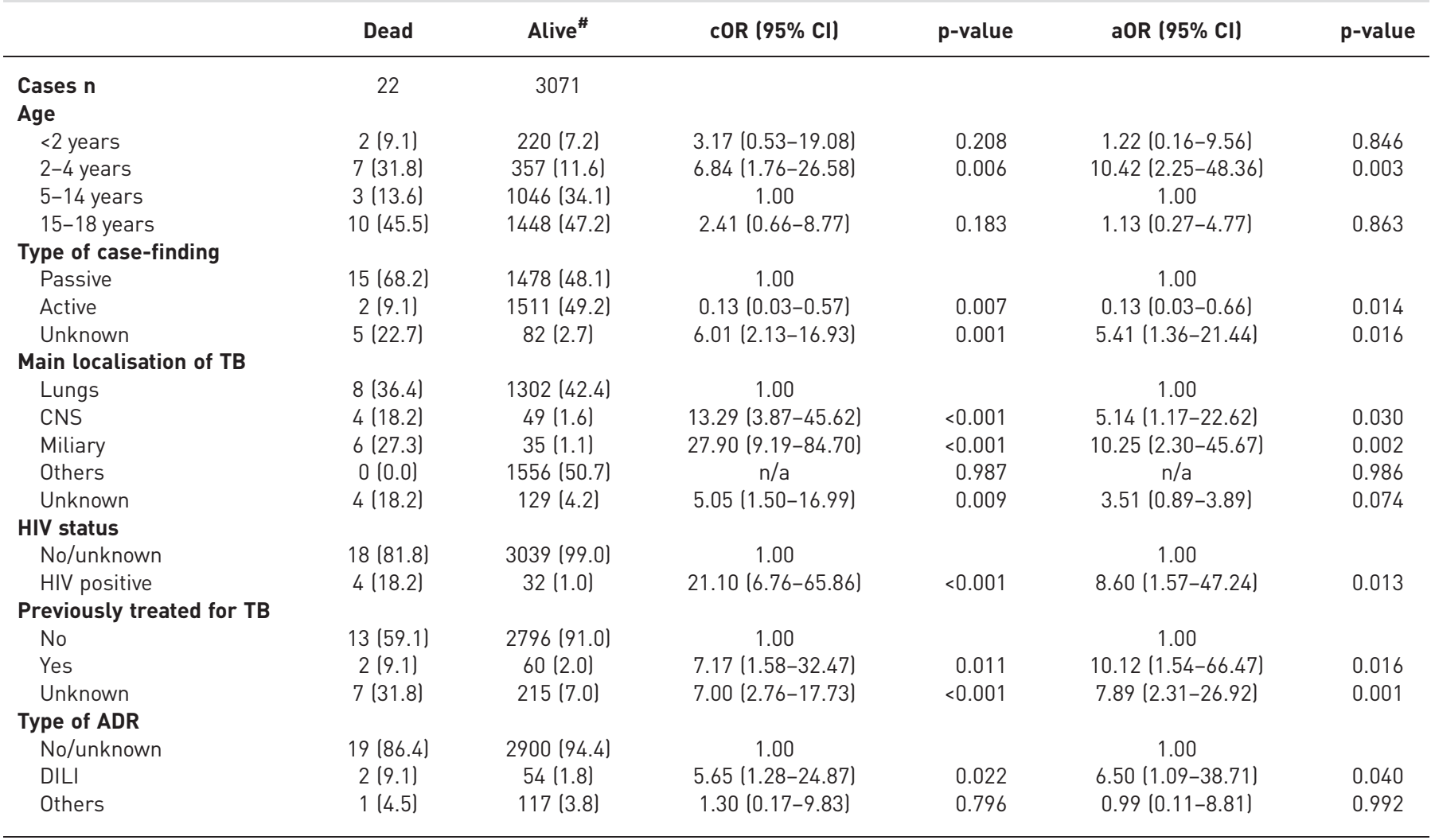

Data are presented as $\mathrm{n}(\%)$, unless otherwise stated. cOR: crude odds ratio; aOR: adjusted odds ratio; CNS: central nervous system; ADR: adverse drug reaction; DILI: drug-induced liver injury; n/a: not applicable. Hosmer-Lemeshow test $p=0.976$; area under the receiver operating characteristic curve 0.96 (95\% Cl 0.94-0.99). \#: included patients who achieved cure or completed treatment and excluded those who were lost to follow-up or with unknown outcomes.

The following factors were associated with higher odds of unfavourable outcome: children aged $<5$ years (aOR 1.58, 95\% CI 1.02-2.46), adolescence (aOR 1.56, 95\% CI 1.11-2.19), illegal immigrants (aOR 5.10, 95\% CI 2.15-12.10), unknown history of TB contact (aOR 2.00, 95\% CI 1.30-3.07), miliary TB (aOR 3.37, 95\% CI 1.42-8.03), multiple ADRs (aOR 7.54, 95\% CI 3.56-15.99) and treatment interruption lasting $>14$ days (aOR 4.90, 95\% CI 2.10-11.42). Treatment supervision by PHNs was associated with lower odds of unfavourable outcome (aOR 0.08, 95\% CI 0.05-0.15) (table 5). Results of the univariate analysis for unfavourable outcome are presented in supplementary table S5.

\section{Discussion}

An overall high success rate of $92.0 \%$ in children was recorded in our study, although this included children with unknown outcomes. This is relatively comparable with studies of children from other low-incidence countries such as Australia (89.4\%) and the UK (88.0\%) [19, 20]. A high success rate was also recorded in adolescents: comparable data from other low-incidence countries are lacking. This underlines that adolescents are often neglected in TB surveillance reports $[3,5]$. The low mortality rate of $<1 \%$ in our study is similar to those reported in the UK and Australia [19, 20], but is lower compared to various reports from high-incidence countries in Asia and Africa (3-17\%) [11-14]. Interestingly, a recent study from South Africa also reported $<1 \%$ mortality rate in children; however, this number was probably higher, as children with severe forms of TB admitted to hospital may have died before diagnosis or after diagnosis but prior to recording in the database [10].

Several risk factors of mortality are shown in our study including children aged 2-4 years, CNS TB, miliary TB, HIV co-infection, re-treated TB cases and cases with DILI. Overall, the increased risk of death in children aged $<5$ years is consistent with those reported in a meta-analysis and a modelling study $[6,7]$. However, in contrast to earlier findings from South Africa [10], our results did not confirm the risk of death in a subgroup of children aged $<2$ years. In children aged $<2-3$ years, the progression of primary 
TABLE 4 Final model for factors associated with loss to follow-up (LTFU) in children and adolescents treated for tuberculosis (TB) in the Netherlands

\begin{tabular}{|c|c|c|c|c|c|c|}
\hline & LTFU & Non-LTFU\# & $\operatorname{cOR}(95 \% \mathrm{CI})$ & p-value & aOR $(95 \% \mathrm{CI})$ & p-value \\
\hline Cases $\mathbf{n}$ & 160 & 3071 & & & & \\
\hline Year of diagnosis median (IQR) & 1999 (1995-2003) & 2001 (1997-2009) & $0.94(0.92-0.97)$ & $<0.001$ & $0.94(0.89-0.98)$ & 0.011 \\
\hline$<5$ years & $24(15.0)$ & $577(18.8)$ & $1.28(0.75-2.18)$ & 0.364 & $1.47(0.84-2.59)$ & 0.178 \\
\hline $5-14$ years & 34 (21.3) & $1046(34.1)$ & 1.00 & & 1.00 & \\
\hline $15-18$ years & $102(63.7)$ & $1448(47.2)$ & $2.17(1.46-3.22)$ & $<0.001$ & $1.91(1.25-2.93)$ & 0.003 \\
\hline Yes, duration $<2.5$ years & $71(44.4)$ & $1139(37.1)$ & $1.54(1.07-2.23)$ & 0.021 & $1.15(0.73-1.81)$ & 0.549 \\
\hline Yes, illegal immigrants & $8(5.0)$ & $22(0.7)$ & 8.99 (3.82-21.17) & $<0.001$ & $4.28(1.60-11.42)$ & 0.004 \\
\hline Yes, duration $\geqslant 2.5$ years & $17(10.6)$ & $502(16.3)$ & $0.84(0.48-1.46)$ & 0.533 & $0.59(0.32-1.10)$ & 0.099 \\
\hline Yes, duration unknown & $13(8.1)$ & $147(4.8)$ & $2.19(1.16-4.12)$ & 0.015 & $1.32(0.65-2.66)$ & 0.443 \\
\hline \multicolumn{7}{|l|}{ Area of living } \\
\hline Urban & 59 (36.9) & $854(27.8)$ & $1.52(1.09-2.11)$ & 0.014 & $1.59(1.10-2.29)$ & 0.014 \\
\hline \multicolumn{7}{|l|}{ Drug susceptibility } \\
\hline Confirmed drug-susceptible TB & $16(10.0)$ & $552(18.0)$ & 1.00 & & 1.00 & \\
\hline Presumed drug-susceptible TB & $127(79.4)$ & 2365 (77.0) & $1.85(1.09-3.14)$ & 0.022 & $1.38(0.72-2.64)$ & 0.332 \\
\hline Confirmed drug-resistant TB & $17(10.6)$ & $154(5.0)$ & $3.81(1.88-7.71)$ & $<0.001$ & $2.31(1.05-5.10)$ & 0.038 \\
\hline \multicolumn{7}{|l|}{ Presence of ADR } \\
\hline No/unknown & $130(81.3)$ & $2900(94.4)$ & 1.00 & & 1.00 & \\
\hline Yes, single ADR & 19 (11.9) & $147(4.8)$ & $2.88(1.73-4.80)$ & $<0.001$ & $2.12(1.18-3.83)$ & 0.012 \\
\hline Yes, multiple ADRs & $11(6.9)$ & $24(0.8)$ & $10.22(4.90-21.32)$ & $<0.001$ & $7.84(3.55-17.33)$ & $<0.001$ \\
\hline \multicolumn{7}{|l|}{ Treatment interruption $>14$ days } \\
\hline No & 20 (12.5) & $1045(34.0)$ & 1.00 & & 1.00 & \\
\hline Yes & $10(6.3)$ & $42(1.4)$ & $12.44(5.48-28.23)$ & $<0.001$ & $6.93(2.72-17.63)$ & $<0.001$ \\
\hline Unknown & $130(81.3)$ & $1984(64.6)$ & $3.42(2.13-5.51)$ & $<0.001$ & 1.03 (0.45-2.35) & 0.938 \\
\hline \multicolumn{7}{|l|}{ Supervision by PHNs } \\
\hline
\end{tabular}

Data are presented as $\mathrm{n}(\%)$, unless otherwise stated. cOR: crude odds ratio; aOR: adjusted odds ratio; IQR: interquartile range; ADR: adverse drug reaction; PHNs: public health nurses. Hosmer-Lemeshow test $p=0.745$; area under the receiver operating characteristic curve 0.75 ( $95 \%$ $\mathrm{Cl}$ 0.72-0.79). \#: included patients who achieved cure or completed treatment and excluded those who died or with unknown outcomes; ๆ: the Hague, Utrecht (city), Amsterdam and Rotterdam; ${ }^{+}$: Groningen, Friesland, Zeeland, Drenthe, Overijssel, Gelderland, Zuid-Holland, Limburg, Utrecht, Noord-Holland, Noord-Brabant, Flevoland or other areas.

infection into severe disease (CNS or miliary TB) is more frequent [21]. These severe forms of disease were associated with mortality in our study, independent to the age of the patients. In high-incidence countries, BCG vaccination has been reported as a highly cost-effective intervention to prevent CNS TB and miliary TB [22]. In the Netherlands, BCG vaccination is only targeted to newborns with a parent coming from a country with estimated $\mathrm{TB}$ incidence $>50$ per 100000 population, and offered to immigrants aged $<12$ years with no evidence of BCG vaccination at pre-entry TB screening [23]. Notably, more patients with severe disease in our study were not BCG-vaccinated: half of them were aged $<5$ years. These results support the previous recommendation by ERKENs et al. [24] to improve the coverage of BCG vaccination among eligible risk groups in the Netherlands.

The role of TB/HIV co-infection as a predictor of mortality in children is supported by various studies, mostly from HIV-endemic settings [10-13]. For TB/HIV co-infected children taking ART, the risk of death is lower than children without ART [6]. In our cohorts, ART status was not completely clear, because the Netherlands Tuberculosis Register has only recorded it since 2016. Next, a recurrent episode of TB can be due to endogenous reactivation of indolent mycobacteria (relapse) or exogenous re-infection, and the latter can be caused by MDR M. tuberculosis strains [25]. Two patients with recurrent TB who died in our study were classified as non-relapse patients, one of which was treated for MDR-TB. It is possible that MDR-TB plays a role in increasing the risk of mortality in recurrent cases. 
TABLE 5 Final model for factors associated with unfavourable outcome in children and adolescents treated for tuberculosis (TB) in the Netherlands

\begin{tabular}{|c|c|c|c|c|c|c|}
\hline & Unfavourable ${ }^{\#}$ & Favourable ${ }^{\pi}$ & COR $(95 \%$ CI $)$ & p-value & aOR $(95 \% \mathrm{CI})$ & p-value \\
\hline Cases n & 325 & 3071 & & & & \\
\hline \multicolumn{7}{|l|}{ Age } \\
\hline$<5$ years & $61(16.8)$ & $577(18.8)$ & $1.38(0.98-1.96)$ & 0.069 & $1.58(1.02-2.46)$ & 0.040 \\
\hline $5-14$ years & $80(24.6)$ & 1046 (34.1) & 1.00 & & 1.00 & \\
\hline $15-18$ years & $184(56.6)$ & $1448(47.2)$ & $1.66(1.26-2.19)$ & $<0.001$ & $1.56(1.11-2.19)$ & 0.010 \\
\hline \multicolumn{7}{|c|}{ Immigrants or asylum seekers } \\
\hline No & $98(30.2)$ & $1261(41.1)$ & 1.00 & & 1.00 & \\
\hline Yes, duration $<2.5$ years & $134(41.2)$ & $1139(37.1)$ & $1.51(1.15-1.99)$ & 0.003 & $1.09(0.74-1.59)$ & 0.663 \\
\hline Yes, illegal immigrants & $17(5.2)$ & $22(0.7)$ & $9.94(5.11-19.34)$ & $<0.001$ & $5.10(2.15-12.10)$ & $<0.001$ \\
\hline Yes, duration $\geqslant 2.5$ years & $37(11.4)$ & 502 (16.3) & $0.95(0.64-1.40)$ & 0.791 & $0.74(0.45-1.20)$ & 0.222 \\
\hline Yes, duration unknown & $39(12.0)$ & $147(4.8)$ & $3.41(2.27-5.14)$ & $<0.001$ & $1.71(0.99-2.96)$ & 0.054 \\
\hline \multicolumn{7}{|l|}{ Known TB contact history } \\
\hline No & 274 (84.3) & $2090(68.1)$ & $2.52(1.85-3.43)$ & $<0.001$ & $2.00(1.30-3.07)$ & 0.002 \\
\hline Yes & $51(15.7)$ & $981(31.9)$ & 1.00 & & 1.00 & \\
\hline \multicolumn{7}{|l|}{ Main localisation of TB } \\
\hline Primary TB infection & $30(9.2)$ & $673(21.9)$ & $0.54(0.36-0.82)$ & 0.004 & $0.85(0.52-1.41)$ & 0.534 \\
\hline Lungs & $107(32.9)$ & $1302(42.4)$ & 1.00 & & 1.00 & \\
\hline Respiratory tract & $18(5.5)$ & $388(12.6)$ & $0.56(0.34-0.94)$ & 0.029 & $0.67(0.39-1.15)$ & 0.146 \\
\hline CNS & $7(2.2)$ & $49(1.6)$ & $1.74(0.77-3.93)$ & 0.184 & $1.52(0.60-3.87)$ & 0.382 \\
\hline Abdominal & $6(1.8)$ & 51 (1.7) & $1.43(0.60-3.41)$ & 0.418 & $1.81(0.72-4.53)$ & 0.207 \\
\hline Osteoarticular & $6(1.8)$ & $67(2.2)$ & $1.09(0.46-2.57)$ & 0.844 & $1.25(0.51-3.05)$ & 0.629 \\
\hline Other organs & $33(10.2)$ & 377 (12.3) & $1.06(0.71-1.60)$ & 0.761 & $0.83(0.53-1.31)$ & 0.427 \\
\hline Miliary & $9(2.8)$ & $35(1.1)$ & $3.13(1.46-6.68)$ & 0.003 & $3.37(1.42-8.03)$ & 0.006 \\
\hline Unknown & $109(33.5)$ & $129(4.2)$ & $10.28(7.45-14.19)$ & $<0.001$ & $3.99(2.56-6.20)$ & $<0.001$ \\
\hline \multicolumn{7}{|l|}{ Previously treated for TB } \\
\hline No & 273 (84.0) & 2796 (91.0) & 1.00 & & 1.00 & \\
\hline Yes & $13(4.0)$ & $60(2.0)$ & $2.22(1.20-4.09)$ & 0.011 & $2.04(0.98-4.24)$ & 0.057 \\
\hline Unknown & $39(12.0)$ & $215(7.0)$ & $1.86(1.29-2.67)$ & 0.001 & $1.07(0.67-1.71)$ & 0.765 \\
\hline \multicolumn{7}{|l|}{ Presence of ADR } \\
\hline No/unknown & $291(89.5)$ & $2900(94.4)$ & 1.00 & & 1.00 & \\
\hline Yes, single ADR & $20(6.2)$ & $147(4.8)$ & $1.36(0.84-2.20)$ & 0.216 & 1.57 (0.89-2.77) & 0.120 \\
\hline Yes, multiple ADRs & $14(4.3)$ & $24(0.8)$ & $5.81(2.97-11.36)$ & $<0.001$ & $7.54(3.56-15.99)$ & $<0.001$ \\
\hline \multicolumn{7}{|c|}{ Treatment interruption $>14$ days } \\
\hline No & 42 (12.9) & $1045(34.0)$ & 1.00 & & 1.00 & \\
\hline Yes & $11(3.4)$ & $42(1.4)$ & 6.52 (3.13-13.55) & $<0.001$ & $4.90(2.10-11.42)$ & $<0.001$ \\
\hline Unknown & $272(83.7)$ & $1984(64.6)$ & $3.41(2.44-4.76)$ & $<0.001$ & $1.58(1.09-2.92)$ & 0.016 \\
\hline \multicolumn{7}{|l|}{ Hospitalised $\geqslant 1$ week } \\
\hline No/<1 week & 238 (73.2) & $2153(70.1)$ & 1.00 & & 1.00 & \\
\hline Yes & $68(20.9)$ & $815(26.5)$ & $0.75(0.57-1.00)$ & 0.050 & $0.71(0.51-1.01)$ & 0.055 \\
\hline Unknown & $19(5.8)$ & $103(3.4)$ & $1.67(1.01-2.77)$ & 0.048 & $0.86(0.46-1.60)$ & 0.633 \\
\hline \multicolumn{7}{|l|}{ Supervised by PHNs } \\
\hline No & 32 (9.8) & $34(1.1)$ & 1.00 & & 1.00 & \\
\hline Yes & $192(59.1)$ & 2976 (96.9) & $0.07(0.04-0.11)$ & $<0.001$ & $0.08(0.05-0.15)$ & $<0.001$ \\
\hline Unknown & $101(31.1)$ & $61(2.0)$ & $1.76(0.99-3.14)$ & 0.055 & $1.01(0.51-2.00)$ & 0.997 \\
\hline
\end{tabular}

Data are presented as $\mathrm{n}(\%)$, unless otherwise stated. cOR: crude odds ratio; aOR: adjusted odds ratio; CNS: central nervous system; ADR: adverse drug reaction; PHNs: public health nurses. Hosmer-Lemeshow test $p=0.506$; area under the receiver operating characteristic curve $0.81(95 \% \mathrm{Cl}$ 0.78-0.84). "\#: the sum of patients who died, were lost to follow-up, or with unknown outcomes; ": the sum of patients who achieved cure or completed treatment.

DILI is one of the most frequent and serious ADRs during TB therapy [26], and has been reported as a predictor of prolonged TB treatment in a Dutch setting [27]. Although a relatively lower rate of DILI was found in our study (1.8\%) compared to other studies of children in Japan (8.1\%) and Indonesia (15\%) $[28,29]$, its clinical implication in increasing the risk of mortality should be taken seriously. Two studies from India and the UK reported DILI as a contributing cause of death in adult TB patients [30,31], with the risk of mortality being even higher if accompanied by jaundice, ascites or encephalopathy [31]. Based on the current WHO guidelines for children, regular monitoring of liver function tests during TB therapy is not mandatory and only recommended if liver tenderness, hepatomegaly, jaundice or early onset of vomiting occur during treatment [32]. Given that severe hepatotoxicity can develop in patients with 
asymptomatic DILI [33], regular monitoring of liver function tests as suggested for adults undergoing TB therapy might also benefit children, to improve treatment outcomes and to prevent mortality.

In the Netherlands, pre-entry LTBI screening is carried out for every immigrant and asylum seeker aged $<18$ years, and radiographic screening only in children aged 12-17 years from a country with TB incidence $\geqslant 100$ per 100000 population was recently suggested [34]. The active case-finding interventions, such as screening for immigrants and asylum seekers as well as source and contact investigations have proven useful in our study to prevent mortality compared to passive case-finding. Given that unknown history of TB contact was found as a risk factor of either patient's or doctor's delay, this highlights the benefits of advocating active case-finding for early diagnosis and early treatment (including preventive therapy), in order to prevent deterioration of the disease. A large randomised controlled trial from Vietnam supports that active case-finding is a cost-effective intervention to increase TB case detection and to reduce all-cause mortality $[35,36]$. In addition, a modelling study reported that household contact investigations could substantially prevent both TB cases and mortality in children [37].

For LTFU, our study identifies the following risk factors: adolescent age, illegal immigrants, unknown history of TB contact, urban domicile, confirmed drug-resistant TB, presence of ADRs and treatment interruption lasting $>14$ days. The increased risk of LTFU in adolescents particularly in male foreign-born adolescents might be due to the lack of awareness of the special needs of this population [15]. To improve adherence in adolescents, appropriate interventions should be understood by considering their developmental and psychosocial issues, tailoring the treatment regimen and ensuring peer and family supports [38]. Implementation of directly observed therapy in our study was not statistically significant to prevent LTFU, even in a particular subgroup of adolescents. This is supported by a meta-analysis that poor adherence in TB treatment cannot be resolved by the directly observed therapy intervention [39]. A recently published randomised controlled trial from the UK reported that smartphone-enabled video-observed therapy is more effective, preferable and cheaper than directly observed therapy for TB treatment observation [40]. In the Netherlands where internet connectivity is not an issue, video-observed therapy might also be relevant as an alternative to directly observed therapy, particularly for adolescents who have a high mobile/internet engagement. A new framework of digital health (e-health), as currently recommended by the WHO [41], might also benefit to ensure treatment adherence. Even though this e-health system has not been widely used in the Netherlands [23], it has great potential as a more patient-friendly intervention for therapy monitoring, particularly for high-risk groups and other individuals with complex confounders (e.g. patients living in urban areas or treated for drug-resistant TB).

Since 2005, a central web-based TB surveillance system was introduced and laboratory data were matched with the Netherlands Tuberculosis Register in real-time. These improvements might have contributed to the reduced number of LTFU cases in recent years. This is supported by our results that most of the LTFU cases (79\%) were notified before 2005. The improved TB outcomes might also be related to the large number of stakeholders involved in TB control activities; from the MPHS, KNCV, RIVM and other health professionals such as pulmonary physicians, paediatricians, TB control physicians, medical microbiologists, medical technicians and PHNs [23]. Our study confirms that treatment supervision by PHNs is a protective factor of LTFU as well as an unfavourable outcome.

A particular strength of this study is a relatively wide range of variables included in the analysis from demographics to disease notification-, clinical-, bacteriological- and treatment-related factors. However, our study has several limitations that should be acknowledged. Due to the retrospective nature of the study using routine data, patient records were partly incomplete for some of the variables. Even though notification of TB is mandatory, the possibility of under-notification cases cannot be ruled out. Through a capture-recapture analysis, the adjusted under-notification of TB in 1998 was estimated to be 7.3\% [42]. However, the completeness of notification is expected to have increased since 2005, when improvements were made to the Netherlands Tuberculosis Register. The high proportion of patients with presumed drug-susceptible TB in our cohorts can be explained by these changes, given that 1139 (99\%) of 1150 patients with culture-confirmed disease but without information on DST results were registered before 2005. Next, our database cannot distinguish between TB contact history with an infectious drug-resistant TB and drug-susceptible TB case, and this may have led to misclassification of presumed drug-susceptible TB in some patients who should have been classified as presumed drug-resistant TB. The low proportion of patients who died and were LTFU in our cohorts could also limit the statistical power of the study. Although the definition of mortality used in this study has followed the current WHO guidelines as all-cause mortality before starting or during the course of treatment [18], the differentiation of death due to TB from other causes along with post mortem evidence could provide a more accurate characterisation of TB-related mortality. In addition, given the details on liver function tests and clinical features of DILI are not registered in the Netherlands Tuberculosis Register, further classification of symptomatic versus asymptomatic DILI cannot be presented. 
In conclusion, this study demonstrates a high rate of successful treatment outcome in children and adolescents treated for TB in the Netherlands from 1993 to 2018. Specific risk groups for mortality, LTFU and unfavourable outcome have been identified for further development of early interventions to support these patients once diagnosed with TB.

Acknowledgements: We would like to thank Henrieke Schimmel (RIVM, Bilthoven, the Netherlands) for her assistance during research approval from the research committee of the Netherlands Tuberculosis Register, and for sending the dataset. We would also like to thank Job van Boven, Anita Faber-Wildeboer and Fatemah Akbari (University of Groningen, Groningen, the Netherlands) for constructive discussions and comments on the manuscript.

Author contributions: F. Gafar, N. van't Boveneind-Vrubleuskaya, B. Wilffert and J-W.C. Alffenaar contributed to conception and design of the study. F. Gafar undertook data extraction and performed data analysis. F. Gafar, N. van't Boveneind-Vrubleuskaya, O.W. Akkerman, B. Wilffert and J-W.C. Alffenaar interpreted the data. F. Gafar and N. van't Boveneind-Vrubleuskaya drafted the manuscript. B. Wilffert and J-W.C. Alffenaar supervised the entire project. All authors critically revised the manuscript for important intellectual content and approved the final version of the manuscript.

Conflict of interest: None declared.

Support statement: This study was supported by the University of Groningen and by the Indonesia Endowment Fund for Education (LPDP), which were in no way involved in study design, writing or reviewing of the manuscript. Funding information for this article has been deposited with the Crossref Funder Registry.

\section{References}

1 World Health Organization (WHO). Global Tuberculosis Report 2018. Geneva, WHO, 2018. www.who.int/tb/ publications/global_report/en/ Date last accessed: July 9, 2019.

2 Snow KJ, Sismanidis C, Denholm J, et al. The incidence of tuberculosis among adolescents and young adults: a global estimate. Eur Respir J 2018; 51: 1702352.

3 García-Basteiro AL, Schaaf HS, Diel R, et al. Adolescents and young adults: a neglected population group for tuberculosis surveillance. Eur Respir J 2018; 51: 1800176.

4 World Health Organization (WHO). Roadmap for childhood tuberculosis. Geneva, WHO, 2013. www.who.int/tb/ areas-of-work/children/roadmap/en/

5 World Health Organization (WHO). Roadmap towards ending TB in children and adolescents, second edition. Geneva, WHO, 2018. www.who.int/tb/publications/2018/tb-childhoodroadmap/en/ Date last accessed: July 9, 2019.

6 Jenkins HE, Yuen CM, Rodriguez CA, et al. Mortality in children diagnosed with tuberculosis: a systematic review and meta-analysis. Lancet Infect Dis 2017; 17: 285-295.

7 Dodd PJ, Yuen CM, Sismanidis C, et al. The global burden of tuberculosis mortality in children: a mathematical modelling study. Lancet Glob Health 2017; 5: e898-e906.

8 Lönnroth K, Migliori GB, Abubakar I, et al. Towards tuberculosis elimination: an action framework for low-incidence countries. Eur Respir J 2015; 45: 928-952.

9 Gröschel MI, van den Boom M, Migliori GB, et al. Prioritising children and adolescents in the tuberculosis response of the WHO European Region. Eur Respir Rev 2019; 28: 180106.

10 Osman M, Lee K, Du Preez K, et al. Excellent treatment outcomes in children treated for tuberculosis under routine operational conditions in Cape Town, South Africa. Clin Infect Dis 2017; 65: 1444-1452.

11 Onyango DO, Yuen CM, Masini E, et al. Epidemiology of pediatric tuberculosis in Kenya and risk factors for mortality during treatment: a national retrospective cohort study. J Pediatr 2018; 201: 115-121.

12 Russell GK, Merle CS, Cooke GS, et al. Towards the WHO target of zero childhood tuberculosis deaths: an analysis of mortality in 13 locations in Africa and Asia. Int J Tuberc Lung Dis 2013; 17: 1518-1523.

13 Hailu D, Abegaz WE, Belay M. Childhood tuberculosis and its treatment outcomes in Addis Ababa: a 5-years retrospective study. BMC Pediatr 2014; 14: 61.

14 Harries AD, Hargreaves NJ, Graham SM, et al. Childhood tuberculosis in Malawi: nationwide case-finding and treatment outcomes. Int J Tuberc Lung Dis 2002; 6: 424-431.

15 Blok N, van den Boom M, Erkens C, et al. Variation in policy and practice of adolescent tuberculosis management in the WHO European Region. Eur Respir J 2016; 48: 943-946.

16 National Institute for Public Health and the Environment (RIVM). Osiris-NTR Tuberculose ziekte: Vragenlijst en handleiding 2017 [Osiris-NTR Tuberculosis Disease: Questionnaire and Manual 2017]. www.rivm.nl/documenten/ osiris-ntr-ziekte-vragenlijst-2017 Date last accessed: July 9, 2019.

17 Nederlandse Vereniging van Artsen voor Longziekten en Tuberculose (NVALT). Richtlijn Medicamenteuze Behandeling van Tuberculose [Guideline on medicinal treatment of tuberculosis]. 2014. www.kncvtbc.org/ uploaded/2015/09/3.4_tuberculose.pdf Date last accessed: July 9, 2019.

18 World Health Organization (WHO). Definitions and Reporting Framework for Tuberculosis - 2013 Revision. Geneva, WHO, 2013. www.who.int/tb/publications/definitions/en/ Date last accessed: August 14, 2019.

19 Abubakar I, Laundy MT, French CE, et al. Epidemiology and treatment outcome of childhood tuberculosis in England and Wales: 1999-2006. Arch Dis Child 2008; 93: 1017-1021.

20 Teo SS, Tay EL, Douglas P, et al. The epidemiology of tuberculosis in children in Australia, 2003-2012. Med J Aust 2015; 203: 440.

21 Marais BJ, Schaaf HS. Tuberculosis in children. Cold Spring Harb Perspect Med 2014; 4: a017855.

22 Trunz BB, Fine P, Dye C. Effect of BCG vaccination on childhood tuberculous meningitis and miliary tuberculosis worldwide: a meta-analysis and assessment of cost-effectiveness. Lancet 2006; 367: 1173-1180. 
23 de Vries G, Riesmeijer R. National Tuberculosis Control Plan 2016-2020: towards elimination. Bilthoven, National Institute for Public Health and the Environment, 2016. www.rivm.nl/bibliotheek/rapporten/2016-0012.pdf Date last accessed: August 14, 2019.

24 Erkens CG, de Vries G, Keizer ST, et al. The epidemiology of childhood tuberculosis in the Netherlands: still room for prevention. BMC Infect Dis 2014; 14: 295.

25 van Rie A, Warren R, Richardson M, et al. Exogenous reinfection as a cause of recurrent tuberculosis after curative treatment. N Engl J Med 1999; 341: 1174-1179.

26 Yee D, Valiquette C, Pelletier M, et al. Incidence of serious side effects from first-line antituberculosis drugs among patients treated for active tuberculosis. Am J Respir Crit Care Med 2003; 167: 1472-1477.

27 van't Boveneind-Vrubleuskaya N, Daskapan A, Kosterink JGW, et al. Predictors of prolonged TB treatment in a Dutch outpatient setting. PLoS One 2016; 11: e0166030.

28 Gafar F, Arifin H, Jurnalis YD, et al. Antituberculosis drug-induced liver injury in children: incidence and risk factors during the two-month intensive phase of therapy. Pediatr Infect Dis J 2019; 38: 50-53.

29 Ohkawa K, Hashiguchi M, Ohno K, et al. Risk factors for antituberculous chemotherapy-induced hepatotoxicity in Japanese pediatric patients. Clin Pharmacol Ther 2002; 72: 220-226.

30 Abbara A, Chitty S, Roe JK, et al. Drug-induced liver injury from antituberculous treatment: a retrospective study from a large TB centre in the UK. BMC Infect Dis 2017; 17: 231.

31 Devarbhavi H, Singh R, Patil M, et al. Outcome and determinants of mortality in 269 patients with combination anti-tuberculosis drug-induced liver injury. J Gastroenterol Hepatol 2013; 28: 161-167.

32 World Health Organization (WHO). Guidance for National Tuberculosis Programmes on the Management of Tuberculosis in Children (2nd ed). Geneva, WHO, 2014. www.who.int/tb/publications/childtb_guidelines/en/ Date last accessed: July 9, 2019.

33 Shang P, Xia Y, Liu F, et al. Incidence, clinical features and impact on anti-tuberculosis treatment of anti-tuberculosis drug induced liver injury (ATLI) in China. PLoS One 2011; 6: e21836.

34 Wolters BA, Akkerman OW, Aartsma Y, et al. Impact of radiographic screening of $>34000$ asylum seeker children. Eur Respir J 2019; 54: 1900579.

35 Fox GJ, Nhung N V, Sy DN, et al. Household-contact investigation for detection of tuberculosis in Vietnam. N Engl J Med 2018; 378: 221-229.

36 Lung T, Marks GB, Nhung NV, et al. Household contact investigation for the detection of tuberculosis in Vietnam: economic evaluation of a cluster-randomised trial. Lancet Glob Health 2019; 7: e376-e384.

37 Dodd PJ, Yuen CM, Becerra MC, et al. Potential effect of household contact management on childhood tuberculosis: a mathematical modelling study. Lancet Glob Health 2018; 6: e1329-e1338.

38 Taddeo D, Egedy M, Frappier JY. Adherence to treatment in adolescents. Paediatr Child Health 2008; 13: 19-24.

39 Karumbi J, Garner P. Directly observed therapy for treating tuberculosis. Cochrane Database Syst Rev 2015; 29: CD003343.

40 Story A, Aldridge RW, Smith CM, et al. Smartphone-enabled video-observed versus directly observed treatment for tuberculosis: a multicentre, analyst-blinded, randomised, controlled superiority trial. Lancet 2019; 393: 1216-1224.

41 Falzon D, Timimi H, Kurosinski P, et al. Digital health for the End TB Strategy: developing priority products and making them work. Eur Respir J 2016; 48: 29-45.

42 van Hest NAH, Smit F, Baars HM, et al. Completeness of notification of tuberculosis in the Netherlands: how reliable is record-linkage and capture-recapture analysis? Epidemiol Infect 2007; 135: 1021-1029. 